\title{
Sacks forcing, Laver forcing, and Martin's Axiom ${ }^{1}$
}

\author{
Haim Judah, Arnold W. Miller², Saharon Shelah
}

\begin{abstract}
In this paper we study the question assuming $\mathrm{MA}+\neg \mathrm{CH}$ does Sacks forcing or Laver forcing collapse cardinals? We show that this question is equivalant to question of what is the additivity of Marczewski's ideal $s^{0}$. We give a proof that it is consistent that Sacks forcing collapses cardinals. On the other hand we show that Laver forcing does not collapse cardinals.
\end{abstract}

\section{Introduction}

Let $\mathbb{S}$ be Sacks perfect set forcing [32]; $p \in \mathbb{S}$ iff $p \subseteq 2^{<\omega}$ is a nonempty subtree and for every $s \in p$ there exists $t \supseteq s$ such that $t^{\wedge} 0 \in p$ and $t^{\wedge} 1 \in p$. The ordering is defined by $p \leq q$ iff $p \subseteq q$. Define $[p]=\left\{x \in 2^{\omega}: \forall n x\lceil n \in p\}\right.$.

The $s^{0}$ ideal of subsets of $2^{\omega}$ is defined by $X \in s^{0}$ iff for every $p \in \mathbb{S}$ there exists $q \leq p$ with $X \cap[q]=\emptyset$. Define $\operatorname{add}\left(s^{0}\right)=\min \left\{|F|: F \subseteq s^{0}, \bigcup F \notin s^{0}\right\}$.

Marczewski's ideal $s^{0}$, which first appeared in [24], has been studied by a number of authors, Aniszczyk, Frankiewicz, Plewik [4], Brown [11][12][13], Brown, Cox [14], Brown, Prikry [15], Corazza [16], Morgan [29], and Pawlikowski [31]. Aniszczyk [5] has asked if MA implies that the ideal $s^{0}$ is $\mathfrak{c}$-additive, i.e., is it true that the union of fewer than continuum many $s^{0}$ sets is an $s^{0}$ set, i.e., $\operatorname{add}\left(s^{0}\right)=\mathfrak{c}$. It is a folklore result that assuming the proper forcing axiom the ideal $s^{0}$ is c-additive (see Abraham [1]). It is also an easy exercise to show the consistency of $\operatorname{add}\left(s^{0}\right)=\omega_{1}$ plus the continuum is large. This happens in the Cohen real model.

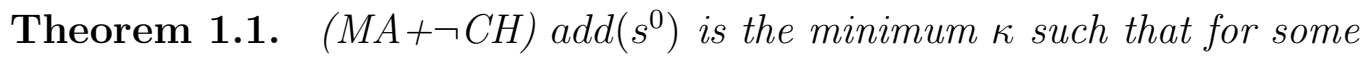
$p \in \mathbb{S}$ we have $p \vdash_{\mathbb{S}} \operatorname{cof}(\mathfrak{c})=\kappa$.

This means that the question of the additivity of the $s^{0}$ ideal is the same as the question of whether Sacks forcing collapses cardinals. In the proof

\footnotetext{
${ }^{1}$ in Archive for Mathematical Logic, 31(1992), 145-161.

${ }^{2}$ Research partially supported by NSF grant 8801139 .
} 
we only use that $\mathfrak{c}$ is regular and MA holds for countable posets. It is well known that Sacks forcing cannot blow up the continuum. In fact Sacks [32] showed that every new element $y$ of $2^{\omega}$ there is a homeomorphism (with perfect domain and range) coded in the ground model which maps the Sacks real to $y$.

Question: Is it consistent with ZFC that $\mathfrak{c}=\omega_{2}$, add $\left(s^{0}\right)=\omega_{1}$, and $\mathbb{S}$ does not collapse $\mathfrak{c}$ ?

Question (Laver): Does $\mathfrak{d}=\aleph_{1}$ imply that $\mathbb{S}$ collapses $\mathfrak{c}$ to $\mathfrak{d}$ ?

The remainder of the section is concerned with three other cardinals associated with the ideal of $s^{0}$-sets. These cardinals have been extensively studied for the ideals of measure and category, see for example Judah and Shelah [19].

$$
\begin{aligned}
& \operatorname{non}\left(s^{0}\right)=\min \left\{|X|: X \notin s^{0}, X \subseteq 2^{\omega}\right\} \\
& \operatorname{cov}\left(s^{0}\right)=\min \left\{|F|: F \subseteq s^{0}, \bigcup F=2^{\omega}\right\} \\
& \operatorname{cof}\left(s^{0}\right)=\min \left\{|F|: F \subseteq s^{0} \text { and } \forall A \in s^{0} \exists B \in F A \subseteq B\right\}
\end{aligned}
$$

Note that $\operatorname{non}\left(s^{0}\right)=\mathfrak{c}$, since any small set of reals is in $s^{0}$.

In Veličković's model $([36])$ he gets MA, $\mathfrak{c}=\omega_{2}$, and $\operatorname{cov}\left(s^{0}\right)=\omega_{1}$. The same is true in the model of Theorem 2.1.

Theorem 1.2. It is relatively consistent with $Z F C$ that $c=\omega_{2}=\operatorname{cov}\left(s^{0}\right)$ and $\operatorname{add}\left(s^{0}\right)=\omega_{1}$.

Fremlin noted that $\operatorname{cof}\left(s^{0}\right)>\mathfrak{c}$. Theorem 1.3 slightly improves this.

Theorem 1.3. $\operatorname{cof}\left(\operatorname{cof}\left(s^{0}\right)\right)>\mathfrak{c}$

This argument also produces an $s^{0}$ set of cardinality $\mathfrak{c}$ (Gurevich and Shelah [18] see also [28]). Pierre Matet [25] has also proved a similar result for $\operatorname{cof}\left(r^{0}\right)$ where $r^{0}$ is the ideal of Ramsey null sets.

So we get (in ZFC) that

$$
\omega_{1} \leq \operatorname{add}\left(s^{0}\right) \leq \operatorname{cov}\left(s^{0}\right) \leq \mathfrak{c}=\operatorname{non}\left(s^{0}\right)<\operatorname{cof}\left(\operatorname{cof}\left(s^{0}\right)\right) \leq \operatorname{cof}\left(s^{0}\right) \leq 2^{\mathfrak{c}} .
$$

The remaining two theorems in this section are easy consistency results to indicate that the inequality

$$
\mathfrak{c}<\operatorname{cof}\left(\operatorname{cof}\left(s^{0}\right)\right) \leq \operatorname{cof}\left(s^{0}\right) \leq 2^{\mathfrak{c}}
$$


is best possible. For simplicity of notation we only consider models of $\mathrm{CH}$.

Theorem 1.4. Let $V \models G C H, \kappa$ any cardinal of cofinality greater than $\omega_{1}$, and let $\mathbb{P}$ be the partial order of countable functions from $\kappa$ to $\omega_{1}$. If $G$ is $\mathbb{P}$-generic over $V$, then $V[G] \models " \omega_{1}=\mathfrak{c}$ and $\operatorname{cof}\left(s^{0}\right)=2^{\omega_{1}}=\kappa$ ". So, for example, it is consistent that $\omega_{2}=\operatorname{cof}\left(\operatorname{cof}\left(s^{0}\right)\right)<\operatorname{cof}\left(s^{0}\right)=\aleph_{\omega_{2}}$.

Theorem 1.5. Let $V$ be a model of $C H$ and $\kappa$ be any regular cardinal with $\omega_{1}<\kappa \leq 2^{\omega_{1}}$. Then there exists a generic extension $V[G]$ (with same cardinals and function $\left.2^{\lambda}\right)$ in which $\operatorname{cof}\left(s^{0}\right)=\kappa$.

In section 2 we show that it is consistent with $\mathrm{MA}+\neg \mathrm{CH}$ that the addivity of the $s^{0}$ ideal is $\omega_{1}$, (equivalently Sacks forcing collapses the continuum.)

Theorem 2.1. It is consistent with $M A+\neg C H$ that the ideal of $s^{0}$ sets is not $\omega_{1}$-additive.

This result was also obtained independently by Velickovic [36] about the same time. Velickovic starts with a model of PFA and forces to kill the additivity of the $s^{0}$ ideal and proves that his forcing does not add any new subsets of $\omega_{1}$.

It is clear from the construction that $\mathfrak{c}$ can be made arbitrarily large, but for simplicity we make it $\omega_{2}$. It is not clear that $\operatorname{add}\left(s^{0}\right)$ can be made anything we want. We assume the reader is familiar with the usual proof of the consistency of MA (see Kunen [21]).

A similar theorem has been proved for Silver forcing by Steprans [35]. For Silver forcing the ideal analagous to $s^{0}$ is the Mycielski ideal [30].

The technique we use is based on an unpublished proof of Kunen who showed that MA $+\neg \mathrm{CH}$ does not settle the existence of $\left(\omega_{1}^{*}, \mathfrak{c}\right)$-gaps or $\left(\mathfrak{c}^{*}, \mathfrak{c}\right)$ gaps. Kunen [22] also uses this argument in his proof that it is possible for $\aleph_{\omega_{1}}$ to be the first cardinal for which MA fails. It has also been used to prove other statements are independent of $\mathrm{MA}+\neg \mathrm{CH}$, see Abraham, Rubin, Shelah [2], Abraham, Todorcevic [3], Devlin [17], and Steprans [35].

Kunen's idea can be summarized as follows. To get the consistency of $\mathrm{MA}+\neg \mathrm{CH}+\mathrm{P}$ where $\mathrm{P}$ is some statement, do a ccc iteration of of small ccc posets. At each step you either force with a ccc poset $\mathrm{Q}$ which preserves the statement $\mathrm{P}$ or you force with a ccc poset $\mathrm{R}$ which destroys the ccc-ness of 
Q but preserves statement $\mathrm{P}$. In either case you have taken care of $\mathrm{Q}$ and preserved statement $\mathrm{P}$.

Section 3 is about Laver forcing $\mathbb{L}$. Conditions in $\mathbb{L}$ are subtrees $p \subseteq \omega^{<\omega}$ with the property that there exists a node $s \in p$ called its root such that:

1. for every $t \in p$ either $t \subseteq s$ or $s \subseteq t$,

2. for every $t \in p$ if $s \subseteq t$, then the set $\operatorname{split}(p, t)={ }^{\operatorname{def}}\left\{n \in \omega: t^{\wedge} n \in p\right\}$ is infinite.

The order relation is given by subset. This forcing was used by Laver [23] to prove the consistency of the Borel conjecture.

Theorem 3.1. Assume MA. Then $\mathbb{L}$ does not collapse cardinals.

Analogously to the $s^{0}$ sets we can define the Laver null sets $l^{0}$ to be all $X \subseteq \omega^{\omega}$ such that for every $p \in \mathbb{L}$ there exists $q \leq p$ with $q \in \mathbb{L}$ such that $[q] \cap X=\emptyset$. It is easy to see that Theorem 3.1 also shows that MA implies that $\operatorname{add}\left(l^{0}\right)=\mathfrak{c}$.

The corresponding theorem for Mathias forcing ([26]) is easy to prove. The fact that the additivity of the ideal of Ramsey null sets is greater than $\omega_{1}$ under MA $+\neg \mathrm{CH}$ plays a key role in Silver's proof [34] that analytic sets are completely Ramsey.

In section 4 we consider a notion of forcing which is half-way in between Sacks forcing $\mathbb{S}$ and Laver forcing $\mathbb{L}$. This forcing is often called superperfect tree forcing or rational perfect set forcing, see Kechris [20], Miller [27], Blass and Shelah [9], Blass [10]

Let $\mathbb{F}$ be superperfect tree forcing which we define as follows. For $p$ a subtree of $\omega^{<\omega}$ define the splitting nodes of $p$ :

$$
\operatorname{splitnodes}(p)=\left\{s \in p: \exists^{\infty} n \in \omega s^{\wedge} n \in p\right\} .
$$

Define $p \in \mathbb{F}$ iff

1. $p$ is a nonempty subtree of $\omega^{<\omega}$,

2. $\operatorname{splitnodes}(p)$ is dense in $p$, i.e., $\forall s \in p \exists t \in \operatorname{splitnodes}(p)$ with $t \supseteq s$, and

3. if any node in $p$ splits it is a splitting node, i.e., if there exists more than one $n \in \omega$ such that $s^{\wedge} n \in p$, then there are infinitely many $n \in \omega$ such that $s^{\wedge} n \in p$. 
Theorem 4.1. Assume MA. Then $\mathbb{F}$ does not collapse cardinals.

As is the case with Ramsey null and Laver null sets, MA implies that $\operatorname{add}\left(f^{0}\right)=\mathfrak{c}$, where $f^{0}$ is the ideal of all subsets $X \subseteq \omega^{\omega}$ such that for every $p \in \mathbb{F}$ there exists $q \leq p$ with $q \in \mathbb{F}$ such that $[q] \cap X=\emptyset$.

Its seems in general that MA can only handle those forcing which have some kind of infinite splitting going on, e.g. Mathias, Laver, superperfect, whereas for those whose conditions are compact, e.g. Sacks, Silver, it is consistent with MA that they collapse the continuum.

In the appendix we solve a problem posed by M. Foreman.

\section{Sacks forcing and Marczewski's ideal}

Let $\mathbb{S}$ be Sacks perfect set forcing, $p \in \mathbb{S}$ iff $p \subseteq 2^{<\omega}$ is a nonempty subtree and for every $s \in p$ there exists $t \supseteq s$ such that $t^{\wedge} 0 \in p$ and $t^{\wedge} 1 \in p$. The ordering is defined by $p \leq q$ iff $p \subseteq q$. Define $[p]=\left\{x \in 2^{\omega}: \forall n x\lceil n \in p\}\right.$.

The $s^{0}$ ideal of subsets of $2^{\omega}$ is defined by $X \in s^{0}$ iff for every $p \in \mathbb{S}$ there exists $q \leq p$ with $X \cap[q]=\emptyset$.

$$
\operatorname{add}\left(s^{0}\right)=\min \left\{|F|: F \subseteq s^{0}, \bigcup F \notin s^{0}\right\}
$$

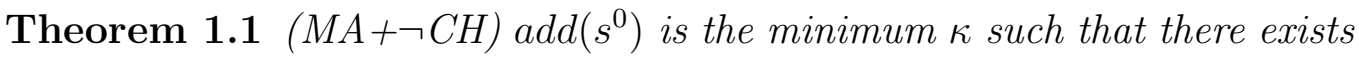
$p \in \mathbb{S}$ such that $p \vdash_{\mathbb{S}} \operatorname{cof}(\mathfrak{c})=\kappa$.

proof:

We will need the following lemma.

Lemma 1.1 Suppose that $D \subseteq \mathbb{S}$ is open and dense, then there exists $A \subseteq D$ a maximal antichain with the property that for every $p \in \mathbb{S}$ if

$[p] \subseteq \bigcup_{q \in A}[q]$, then there exists $A^{\prime} \in[A]^{<\mathfrak{c}}$ such that $[p] \subseteq \bigcup_{q \in A^{\prime}}[q]$.

proof:

Let $\mathbb{S}=\left\{q_{\alpha}: \alpha<\mathfrak{c}\right\}$. Build $A=\left\{p_{\alpha}: \alpha<\mathfrak{c}\right\}$ and $\left\{x_{\alpha}: \alpha<\mathfrak{c}\right\} \subseteq 2^{\omega}$ by induction on $\alpha$. At stage $\alpha$ if $\left[q_{\alpha}\right]$ is not covered by $\bigcup_{\beta<\alpha}\left[p_{\beta}\right]$, then choose $x_{\alpha} \in\left[q_{\alpha}\right] \backslash \bigcup_{\beta<\alpha}\left[p_{\beta}\right]$ otherwise let $x_{\alpha}=x_{0}$. If $q_{\alpha}$ is compatible with some $p_{\beta}$ then let $p_{\alpha}=p_{0}$. Otherwise since a perfect set can be divided into perfectly 
many disjoint perfect sets it is possible to find $p_{\alpha} \leq q_{\alpha}$ such that $\left[p_{\alpha}\right]$ is disjoint from $\left\{x_{\beta}: \beta \leq \alpha\right\}$.

Now suppose $\vdash \operatorname{cof}(\mathfrak{c})=\kappa$. We show $\operatorname{add}\left(s^{0}\right) \leq \kappa$. Let

$$
\vdash_{\mathbb{S}} \text { " } \tau: \kappa \rightarrow \mathfrak{c} \text { is cofinal". }
$$

For each $\alpha<\kappa$ let

$$
D_{\alpha}=\{p \in \mathbb{S}: \exists \beta p \Vdash \tau(\alpha)=\beta\} .
$$

Let $A_{\alpha} \subseteq D_{\alpha}$ be the antichains obtained from the lemma. And let

$$
X_{\alpha}=2^{\omega} \backslash \bigcup_{p \in A_{\alpha}}[p]
$$

Since $A_{\alpha}$ is a maximal antichain it is easy to see that $X_{\alpha}$ is an $s^{0}$ set. Now suppose that $\mathfrak{c}$ is regular and $\kappa<\mathfrak{c}$. We claim that $X=\bigcup_{\alpha<\kappa} X_{\alpha}$ is not an $s^{0}$ set. If it is we must have $[p] \cap X=\emptyset$ for some $p \in \mathbb{S}$. But this implies that $[p] \subseteq \bigcup_{q \in A_{\alpha}}[q]$ for each $\alpha$ so there exists $A_{\alpha}^{\prime} \subseteq A_{\alpha}$ of cardinality less than $\mathfrak{c}$ such that $[p] \subseteq \bigcup_{q \in A_{\alpha}^{\prime}}[q]$. By the definition of $D_{\alpha}$ this means there exists $Y_{\alpha}$ of cardinality less than $\mathfrak{c}$ such that

$$
p \Vdash \forall \alpha<\kappa \tau(\alpha) \in \check{Y}_{\alpha} .
$$

This contradicts the fact that $\tau$ is a cofinal map.

For the other direction suppose that $\operatorname{add}\left(s^{0}\right)=\kappa$ and suppose for contradiction that $\vdash_{\mathbb{S}} \operatorname{cof}(\mathfrak{c})>\kappa$. We can assume $\kappa<\mathfrak{c}$. Let $X_{\alpha}$ be $s^{0}$ sets such that

$$
X=\bigcup_{\alpha<\kappa} X_{\alpha}
$$

is not $s^{0}$. Working below the appropriate $p^{*}$ (namely, some withness to the fact $X \notin s^{0}$ ), we have that every $p$ satisfies $[p] \cap X \neq \emptyset$. Let $D_{\alpha}=\{p \in \mathbb{S}$ : $\left.[p] \cap X_{\alpha}=\emptyset\right\}$. It is easy to see that $D_{\alpha}$ is open dense. Let $A_{\alpha} \subseteq D_{\alpha}$ be any maximal antichain. Let $\mathbb{S}=\left\{p_{\alpha}: \alpha<\mathfrak{c}\right\}$ be listed without repetitions and define an $\mathbb{S}$ name $\tau: \kappa \rightarrow \mathfrak{c}$ by $\tau(\alpha)$ is the unique $\beta$ such that $p_{\beta} \in G \cap A_{\alpha}$ where $G$ is the $\mathbb{S}$-generic filter. Since the cofinality of $\mathfrak{c}$ is greater than $\kappa$ in the extension, we can find $\beta<\mathfrak{c}$ and $p \in \mathbb{S}$ such that

$$
p \Vdash \forall \alpha<\kappa \tau(\alpha)<\beta \text {. }
$$


Let $H=\left\{p_{\alpha}: \alpha<\beta\right\}$. Clearly for every $r \leq p$ and $\alpha<\kappa$ there exists $q \in A_{\alpha} \cap H$ which is compatible with $r$. Define for $s \in p, p_{s}=\{t \in p: s \subseteq$ $t$ or $t \subseteq s\}$. For a fixed $\alpha$, there are two possibilities:

1. for every $q \in A_{\alpha} \cap H$ the set $[q] \cap[p]$ is nowhere dense in $[p]$, or

2. there exists $q \in A_{\alpha} \cap H$ such that for some $s \in p$ we have $p_{s} \leq q$.

But (1) is impossible, since by MA we could find a perfect $r \leq p$ such that for every $q \in A_{\alpha} \cap H$ we have $[r] \cap[q]=\emptyset$. Also (1) is impossible for any $p_{s}$ in place of $p$. Hence we can find $E_{\alpha} \subseteq p$ such that for every $s \in E_{\alpha}$ there exists $q \in A_{\alpha}$ such that $p_{s} \leq q$ and $E_{\alpha}$ is a dense set of nodes in $p$, ie. for every $t \in p$ there exists $s \supseteq t$ with $s \in E_{\alpha}$.

Consider the forcing notion:

$$
\mathbb{P}=\left\{(F, n): \emptyset \neq F \subseteq p \cap 2^{n}\right\}
$$

and ordered by $(F, n) \leq\left(F^{\prime}, n^{\prime}\right)$ iff $n \geq n^{\prime}$ and $F^{\prime}=\left\{s \uparrow n^{\prime}: s \in F\right\}$. Since $\mathbb{P}$ is countable, forcing with it is the same as Cohen real forcing. Given $G$ a $\mathbb{P}$-filter let $r=\bigcup\{F: \exists n(F, n) \in G\}$. It is easy to write down countably many dense sets which will guarantee that $r \in \mathbb{S}$. Also $\kappa$ many dense sets which will make it true that for every $\alpha<\kappa$ there exists $n<\omega$ for every $s \in r \cap 2^{n}$ there exists $q \in A_{\alpha}$ with $r_{s} \leq q$. But this implies that for every $\alpha$

$$
[r] \subseteq \bigcup_{q \in A_{\alpha}}[q]
$$

and so $[r] \cap \bigcup_{\alpha<\kappa} X_{\alpha}=\emptyset$. This contradiction finishes the proof of Theorem 1.1 .

Three other cardinal functions of an ideal $I$ are non, cov, and cof:

$$
\begin{aligned}
& \operatorname{non}(I)=\min \left\{|X|: X \notin I, X \subseteq 2^{\omega}\right\} \\
& \operatorname{cov}(I)=\min \left\{|F|: F \subseteq I, \bigcup F=2^{\omega}\right\} \\
& \operatorname{cof}(I)=\min \{|F|: F \subseteq I \text { and } \forall A \in I \exists B \in F A \subseteq B\}
\end{aligned}
$$

Theorem 1.2 It is relatively consistent with $Z F C$ that $c=\omega_{2}=\operatorname{cov}\left(s^{0}\right)$ and $\operatorname{add}\left(s^{0}\right)=\omega_{1}$.

proof:

The iterated Sacks forcing model is described in Baumgartner-Laver [8]. It is obtained by starting with a ground model which satisfies $\mathrm{CH}$ and then 
iterating $\mathbb{S} \omega_{2}$ times with countable supports. The continuum ends up being $\omega_{2}$ and no cardinals are collapsed.

The fact that $\operatorname{add}\left(s^{0}\right)=\omega_{1}$ follows from half of the proof of Theorem 1.1 and the theorem (proved in [8]) that Sacks forcing over $V_{\omega_{2}}$ collapses $\omega_{2}$.

To see that $\operatorname{cov}\left(s^{0}\right)=\omega_{2}$ let $V_{\alpha}$ for $\alpha \leq \omega_{2}$ be the iteration sequence. Let $\left\langle X_{\alpha}: \alpha<\omega_{1}\right\rangle \in V_{\omega_{2}}$ be $s^{0}$ sets. In $V_{\omega_{2}}$ let $f_{\alpha}: \mathbb{S} \rightarrow \mathbb{S}$ be such that for every $p \in \mathbb{S} f_{\alpha}(p) \leq p$ and $\left[f_{\alpha}(p)\right] \cap X_{\alpha}=\emptyset$. Since the iteration has the $\omega_{2}$ chain condition by a Lowenheim-Skolem argument it is possible to find $\gamma<\omega_{2}$ so that

$$
\left\langle f_{\alpha}\left\lceil\mathbb{S}^{V_{\gamma}}: \alpha<\omega_{1}\right\rangle \in V_{\gamma} .\right.
$$

But, we claim the $\gamma^{\text {th }}$ Sacks real $x_{\gamma}$ is not in $\bigcup_{\alpha<\omega_{1}} X_{\alpha}$. If it was, then for some condition $p \in \mathbb{P}_{\left[\gamma, \omega_{2}\right)}$ and some $\alpha<\omega_{1}$ we would have:

$$
p \Vdash x_{\gamma} \in X_{\alpha} .
$$

But letting $q=p(\gamma) \in \mathbb{S}$ and letting $r(\gamma)=f_{\alpha}(q)$ and $r(\beta)=p(\beta)$ for $\beta>\gamma$ we see that

$$
r \vdash x_{\gamma} \notin X_{\alpha}
$$

Theorem $1.3 \operatorname{cof}\left(\operatorname{cof}\left(s^{0}\right)\right)>\mathfrak{c}$

proof:

Let $\kappa=\operatorname{cof}\left(s^{0}\right)$ and $\left\{Y_{\alpha}: \alpha<\kappa\right\} \subseteq s^{0}$ be a cofinal family. Let

$$
\left\{p_{\alpha}: \alpha<\mathfrak{c}\right\}
$$

be all perfect subsets of $2^{\omega} \times 2^{\omega}$,

$$
\left\{x_{\alpha}: \alpha<\mathfrak{c}\right\}=2^{\omega} \text {, and } L_{\alpha}=\left\{\left(x_{\alpha}, y\right): y \in 2^{\omega}\right\} \text {. }
$$

Build $q_{\alpha} \subseteq L_{\alpha}$ perfect such that for every $\beta<\alpha$ if $p_{\beta} \cap L_{\alpha}$ is countable, then $q_{\alpha}$ is disjoint from $p_{\beta}$. This is easily done since any perfect set splits into $\mathfrak{c}$ many disjoint perfect sets. Now we assume for contradiction that $\operatorname{cof}(\kappa) \leq \mathfrak{c}$. Let $F: \mathfrak{c} \rightarrow \kappa$ have unbounded range. Since $\kappa=\operatorname{cof}\left(s^{0}\right)$ for each $\beta<\mathfrak{c}$ the set

$$
\left\{Y_{\alpha} \cap q_{\beta}: \alpha<F(\beta)\right\}
$$


is not cofinal in the $s^{0}$ subsets of $q_{\beta}$. So there exists an $s^{0}$ set $Z_{\beta} \subseteq q_{\beta}$ such that $Z_{\beta}$ is not covered by any $Y_{\alpha}$ with $\alpha<F(\beta)$. Then $Z=\bigcup_{\beta<\mathrm{c}} Z_{\beta}$ is not covered by any $Y_{\alpha}$, so it suffices to see that $Z$ is an $s^{0}$ set. Let $p \subseteq 2^{\omega} \times 2^{\omega}$ be any perfect set. If $p \cap L_{\beta}$ is uncountable for any $\beta$, then let $q \subseteq p \cap L_{\beta}$ be perfect such that $q \cap Z_{\beta}=\emptyset$, then $q \cap Z=\emptyset$. Otherwise $p \cap L_{\beta}$ is countable for every $\beta$. If $p=p_{\alpha}$, then by construction $q_{\beta} \cap p_{\alpha}=\emptyset$ for every $\beta \geq \alpha$. It follows that

$$
(p \cap Z) \subseteq\left(p \cap \bigcup_{\beta<\alpha} L_{\beta}\right)
$$

But $p \cap\left(\bigcup_{\beta<\alpha} L_{\beta}\right)$ is a set of cardinality less than $\mathfrak{c}$ and so there exists $q \subseteq p$ perfect such that $q \cap\left(\bigcup_{\beta<\alpha} L_{\beta}\right)=\emptyset$, and hence $q \cap Z=\emptyset$.

Theorem 1.4 Let $V \models G C H, \kappa$ any cardinal of cofinality greater than $\omega_{1}$, and let $\mathbb{P}$ be the partial order of countable functions from $\kappa$ to $\omega_{1}$. If $G$ is $\mathbb{P}$-generic over $V$, then $V[G] \models " \omega_{1}=\mathfrak{c}$ and $\operatorname{cof}\left(s^{0}\right)=2^{\omega_{1}}=\kappa$ ". So, for example, it is consistent that $\omega_{2}=\operatorname{cof}\left(\operatorname{cof}\left(s^{0}\right)\right)<\operatorname{cof}\left(s^{0}\right)=\aleph_{\omega_{2}}$.

proof:

Countably closed forcing does not add any reals. By the usual chain condition argument and decomposition as a product forcing it is enough to see:

If $G: \omega_{1} \rightarrow \omega_{1}$ is $\omega_{1}^{<\omega_{1}}$-generic over $V$ then in $V[G]$ there exists an $s^{0}$ set $X$ which is not covered by any $s^{0}$ set in $V$.

By a similar construction as in the proof of Theorem 1.3 we can find in $V$ disjoint perfect sets $q_{\alpha}$ for $\alpha<\omega_{1}=\mathfrak{c}$ such that any $X \subseteq 2^{\omega}$ which meets each $q_{\alpha}$ in a singleton is an $s^{0}$-set. Now just use the generic function $G: \omega_{1} \rightarrow \omega_{1}$ to pick out a single element of each $q_{\alpha}$.

Theorem 1.5 Let $V$ be a model of $C H$ and $\kappa$ be any regular cardinal with $\omega_{1}<\kappa \leq 2^{\omega_{1}}$, then there exists a generic extension $V[G]$ (with same cardinals and function $\left.2^{\lambda}\right)$ in which $\operatorname{cof}\left(s^{0}\right)=\kappa$.

proof:

To bring down $\operatorname{cof}\left(s^{0}\right)$ but leave $2^{\mathfrak{c}}$ large, force with the following partial order $\mathbb{P}$ :

$(X, f) \in \mathbb{P}$ iff 
1. $X \in s^{0}$,

2. $f: \mathbb{S} \rightarrow \mathbb{S}$ is a countable partial function, and

3. $\forall p \in \operatorname{domain}(f) \quad f(p) \leq p$ and $[f(p)] \cap X=\emptyset$.

The ordering on $\mathbb{P}$ is defined by $\left(X^{\prime}, f^{\prime}\right) \leq(X, f)$ iff $X^{\prime} \supseteq X$ and $f^{\prime} \supseteq f$. Clearly $\mathbb{P}$ is countably closed and two elements of $\mathbb{P}$ with the same $f$ are compatible, so it is $\omega_{1}$-centered. It is also true that it is well met, ie. infimums exist.

Now define $\mathbb{P}_{\omega}^{\omega_{1}}$ to be those elements of $p \in \mathbb{P}^{\omega_{1}}$ with countable support, ie. there are at most countably many $\alpha<\omega_{1}$ such that $p(\alpha) \neq(\emptyset, \emptyset)$. It is easy to see that forcing with $\mathbb{P}_{\omega}^{\omega_{1}}$ adds an $\left\langle f_{\alpha}: \alpha<\omega_{1}\right\rangle$ such that if

$$
X_{\alpha}=2^{\omega} \backslash \bigcup_{p \in \mathbb{S}}\left[f_{\alpha}(p)\right]
$$

then each $X_{\alpha} \in s^{0}$ and for every $Y \in s^{0} \cap V$ there exists $\alpha<\omega_{1}$ such that $Y \subseteq X_{\alpha}$. Now just like in the usual proofs of Generalized Martin's Axiom, see [33], [37], or [7], we iterate forcing with $\mathbb{P}_{\omega}^{\omega_{1}} \kappa$ many times with countable supports. Since cofinally often we add a function $f: \omega_{1} \rightarrow \omega_{1}$ which is $\omega_{1}^{<\omega_{1}}$ generic, by the argument of Theorem 1.4 we see that $\operatorname{cof}\left(s^{0}\right) \geq \kappa$. Since the iteration satisfies the $\omega_{2}$ chain condition every subset of $2^{\omega}$ appears at some intermediate stage, and is covered by one of the generic $s^{0}$ sets. Hence $\operatorname{cof}\left(s^{0}\right) \leq \kappa$

\section{Martin's Axiom and Marczewski's ideal}

In this section we start with a model satisfying the continuum hypothesis and by an inductive construction, we will get a model for Martin's Axiom where the Marczewski's ideal, $s^{0}$, is not $\omega_{1}$ additive.

Theorem 2.1 It is consistent with $M A+\neg C H$ that the ideal of $s^{0}$ sets is not $\omega_{1}$-additive.

proof:

Recall that $[T]=\left\{x \in 2^{\omega}: \forall n<\omega x \uparrow n \in T\right\}$.

Define $(*)\left\langle T_{j}: j<\zeta\right\rangle$ where $\zeta \leq \omega_{2}$ and each $T_{j} \in \mathbb{S}$ as follows: 
$(*)\left\langle T_{j}: j<\zeta\right\rangle$ : Given $\left\langle F_{\alpha}: \alpha<\omega_{1}\right\rangle$ pairwise disjoint finite subsets of $\zeta$ there exists $\alpha \neq \beta$ such that $\forall i \in F_{\alpha} \forall j \in F_{\beta}\left[T_{i}\right] \cap\left[T_{j}\right]=\emptyset$.

The Construction: By induction on $\zeta \leq \omega_{2}$ we shall

1. define $\left\langle P_{i}, Q_{j}: j<\zeta, i \leq \zeta\right\rangle$ a finite support iteration of ccc forcing notions with as usual $P_{\zeta+1}=P_{\zeta} * Q_{\zeta}$,

2. define $\left\langle T_{j}: j<\zeta\right\rangle \in V^{P_{\zeta}}$ such that $V^{P_{\zeta}} \models(*)\left\langle T_{j}: j<\zeta\right\rangle$,

3. make sure that $\left\{T_{j}: j<\omega_{2}\right\}$ is dense in $\mathbb{S} \cap V^{P_{\omega_{2}}}$, and

4. make sure that MA holds in $V^{P_{\omega_{2}}}$.

In order to make MA true we list all possible $P_{\omega_{2}}$ names for posets of cardinality $\omega_{1}$ say $R_{\zeta}$ for $\zeta<\omega_{2}$. We then apply Lemma 2.2 to the ground model $V^{P_{\zeta}}$ to get either that forcing with $\mathbb{P}_{\zeta} * R_{\zeta}$ satisfies $(*)\left\langle T_{j}: j<\zeta\right\rangle$ or there exists $Q$ such that forcing with $P_{\zeta} * Q$ satisfies $(*)\left\langle T_{j}: j<\zeta\right\rangle$ and $R_{\zeta}$ does not have ccc. Let $T_{\zeta}^{\prime}$ be any element of $\mathbb{S} \cap V^{P_{\zeta}}$. However make sure that at the end we have $\left\{T_{\zeta}^{\prime}: \zeta<\omega_{2}\right\}$ is dense in $\mathbb{S} \cap V^{P_{\omega_{2}}}$; because $T_{\zeta} \leq T_{\zeta}^{\prime}$ (see $(* *)$ below) this ensures that $\left\{T_{\zeta}: \zeta<\omega_{2}\right\}$ is dense. Note that since $Q_{T_{\zeta}^{\prime}}$ (defined below) is a countable poset it cannot destroy $(*)$ nor can adding one tree destroy $(*)$.

We then let $Q_{\zeta}=R * Q_{T_{\zeta}^{\prime}}$ or $Q_{\zeta}=Q * Q_{T_{\zeta}^{\prime}}$ which ever preserves $(*)$. This concludes the proof of Theorem 2.1.

Lemma 2.1 In $V^{P_{\omega_{2}}}$ the additivity of $s^{0}$ ideal is $\omega_{1}$, in fact $\operatorname{cov}\left(s^{0}\right)=\omega_{1}$.

proof:

Choose $\left\langle T_{i, j} \in \mathbb{S}: i<\omega_{1}, j<\omega_{2}\right\rangle$ such that for each $i, j$ there is a unique $\zeta(i, j)$ such that $T_{i, j} \subseteq T_{\zeta(i, j)}$ and also for each $i<\omega_{1}$

$$
A_{i}=\left\{T_{i, j}: j<\omega_{2}\right\}
$$

is a maximal antichain in $\mathbb{S}$. This is easy to do since the family $\left\{T_{\zeta}: \zeta<\omega_{2}\right\}$ is dense in $\mathbb{S}$ and every condition in $\mathbb{S}$ has a perfect set of incompatible extensions. If

$$
X_{i}=2^{\omega} \backslash \bigcup_{T \in A_{i}}[T]
$$


then each $X_{i}$ is an $s^{0}$-set since $A_{i}$ is a maximal antichain. But $2^{\omega}=\bigcup_{i<\omega_{1}} X_{i}$, since otherwise if $x \in 2^{\omega} \backslash \bigcup_{i<\omega_{1}} X_{i}$, then choosing $F_{\alpha}=\{\zeta(\alpha, \beta)\}$ where $x \in\left[T_{\alpha, \beta}\right] \subseteq\left[T_{\zeta(\alpha, \beta)}\right]$ witnesses the failure of $(*)$.

The following lemma is the key to preserving $(*)$ while at the same time making MA true.

Lemma 2.2 Suppose $V \models$ “(*) $\left\langle T_{j}: j<\zeta\right\rangle, R$ is ccc", and $V^{R} \models " \neg(*)\left\langle T_{j}\right.$ : $j<\zeta\rangle$ ". Then there exists a ccc partial order $Q$ such that $V^{Q} \models "(*)\left\langle T_{j}\right.$ : $j<\zeta\rangle$ and $R$ is not $c c c "$.

proof:

Let $\left\langle F_{\alpha}^{\prime}: \alpha<\omega_{1}\right\rangle$ be $R$-names and $r \in R$ such that

$$
r \mid \vdash_{R} \text { " }\left\langle F_{\alpha}^{\prime}: \alpha<\omega_{1}\right\rangle \text { is a counterexample to }(*) \text { ". }
$$

Let $\left\langle r_{\alpha}, F_{\alpha}: \alpha<\omega_{1}\right\rangle \in V$ be such that each $r_{\alpha} \leq r$ and

$$
r_{\alpha} \Vdash F_{\alpha}^{\prime}=\check{F}_{\alpha}
$$

For some $A \in\left[\omega_{1}\right]^{\omega_{1}}$ we have that $\left\langle F_{\alpha}: \alpha \in A\right\rangle$ is a $\Delta$-system. Note that root of this $\Delta$-system must be empty because if for some $\alpha \neq \beta F_{\alpha} \cap F_{\beta} \neq \emptyset$, then $r_{\alpha}$ and $r_{\beta}$ are incompatible. But $R$ has the ccc.

Define $Q$ to be the set of all $q \in[A]^{<\omega_{0}}$ such that if $\alpha \neq \beta \in q$, then

$$
\forall i \in F_{\alpha} \forall j \in F_{\beta}\left[T_{i}\right] \cap\left[T_{j}\right]=\emptyset .
$$

Order $Q$ by inclusion. Note that if $q \in Q$ and $\alpha, \beta \in q$ we have $r_{\alpha}$ and $r_{\beta}$ are incompatible in $R$. Hence forcing with $Q$ adds an uncountable antichain to $R$. So it is enough to prove the following two claims.

Claim 1. $Q$ satisfies ccc.

proof:

If not let $\left\langle q_{\alpha}: \alpha<\omega_{1}\right\rangle$ be pairwise incompatible. For some $B \in\left[\omega_{1}\right]^{\omega_{1}}$ we have that $\left\langle q_{\alpha}: \alpha \in B\right\rangle$ forms a $\Delta$-system with root $q^{*}$. Now $q_{\alpha} \cup q_{\beta} \in Q$ iff $\left(q_{\alpha} \backslash q^{*}\right) \cup\left(q_{\beta} \backslash q^{*}\right) \in Q$. Therefore without loss of generality we may assume $\left\langle q_{\alpha}: \alpha \in B\right\rangle$ are pairwise disjoint. If we let $K_{\alpha}=\bigcup_{\delta \in q_{\alpha}} F_{\delta}$, then $\left\langle K_{\alpha}: \alpha \in B\right\rangle$ are pairwise disjoint. Therefore by applying $(*)$ in $V$, there are $\alpha \neq \beta \in B$ satisfying

$$
\forall i \in K_{\alpha} \forall j \in K_{\beta}\left[T_{i}\right] \cap\left[T_{j}\right]=\emptyset
$$


and so $q_{\alpha} \cup q_{\beta} \in Q$.

Claim 2. $Q$ preserves $(*)$.

proof:

Let $q \in Q$ and $\left\langle H_{\alpha}^{\prime}: \alpha<\omega_{1}\right\rangle$ be a $Q$-name such that such that

$q \vdash_{Q}$ " $\left\langle H_{\alpha}^{\prime}: \alpha<\omega_{1}\right\rangle$ are pairwise disjoint finite subsets of $\zeta$ ".

Let $\left\langle q_{\alpha}, H_{\alpha}: \alpha<\omega_{1}\right\rangle \in V$ be such that $q_{\alpha} \leq q$ and

$$
q_{\alpha} \Vdash \text { " } H_{\alpha}^{\prime}=\check{H}_{\alpha} " .
$$

Then for some $B \in\left[\omega_{1}\right]^{\omega_{1}}$ the following form $\Delta$-systems: $\left\langle q_{\alpha}: \alpha \in B\right\rangle$ and $\left\langle H_{\alpha}: \alpha \in B\right\rangle$. By ccc of $Q$ we have that the root of $\left\langle H_{\alpha}: \alpha \in B\right\rangle$ must be empty. Let $q^{*}$ be the root of the $q$ 's so $q_{\alpha} \cap q_{\beta}=q^{*}$ for $\alpha \neq \beta \in B$. Now we define

$$
K_{\alpha}=\bigcup_{\delta \in q_{\alpha} \backslash q^{*}} F_{\delta} \cup H_{\alpha} .
$$

Since the $H$ 's and $F$ 's are pairwise disjoint families it is easy to find $C \in[B]^{\omega_{1}}$ such that $K_{\alpha}$ for $\alpha \in C$ are pairwise disjoint.

Hence by $(*)$ in $V$ there exists $\alpha \neq \beta \in C$ such that

$$
\forall i \in K_{\alpha} \forall j \in K_{\beta}\left[T_{i}\right] \cap\left[T_{j}\right]=\emptyset .
$$

But this means that $q_{\alpha} \cup q_{\beta} \in Q$ and

$$
q_{\alpha} \cup q_{\beta} \vdash_{Q} " \forall i \in H_{\alpha}^{\prime} \forall j \in H_{\beta}^{\prime}\left[T_{i}\right] \cap\left[T_{j}\right]=\emptyset " .
$$

Since we started with an arbitrary condition and name we have $(*)$ holds in $V^{Q}$. Hence Claim 3 is proven and this finishes the proof of Lemma 2.2.

Next we show that property $(*)$ is preserved at limit stages. Note that $(*)$ is trivially preserved at stage $\omega_{2}$. The preservation of $(*)$ at stages of cofinality $\omega_{1}$ is more delicate and requires that we specify the details of exactly how we pick the trees $T_{i}$.

For $T^{\prime} \in \mathbb{S}$ let $Q_{T^{\prime}}=\left\{(F, n): \emptyset \neq F \subseteq T^{\prime} \cap 2^{n}\right\}$

Order $Q_{T^{\prime}}$ by $(F, n) \leq(G, m)$ iff $n \geq m$ and $G=\{s\lceil m: s \in F\}$. Forcing with $Q_{T^{\prime}}$ naturally determines a perfect subtree $T$ of $T^{\prime}$ as follows: if $G$ is $Q_{T^{\prime}}$ generic, then let

$$
T=\{s: \exists(F, n) \in G s \in F\} .
$$


In our construction $T_{j}$ is obtained by forcing with $Q_{T^{\prime}}$ for some $T^{\prime}$. Recall that $T_{s}=\{t \in T: s \subseteq t$ or $t \subseteq s\}$. For $T$ which is $Q_{T^{\prime}}$-generic it is easy to see that

1. if $T_{0} \in \mathbb{S}$ is in the ground model, then for some $n<\omega$ for every $s \in 2^{n} \cap T_{0} \cap T \quad T_{s} \subseteq T_{0}$, and

2. for any $p \in Q_{T^{\prime}}$ there exists $n<\omega, t_{0}, t_{1}, q_{0}, q_{1} \leq p$ such that $t_{0}$ and $t_{1}$ are disjoint, $q_{0} \Vdash T \cap 2^{n}=t_{0}$, and $q_{1} \Vdash T \cap 2^{n}=t_{1}$.

Our construction will satisfy the following:

(**) For every $\alpha<\omega_{2} T_{\alpha}$ is $Q_{T_{\alpha}^{\prime}}$-generic over $V^{P_{\alpha}}$ for some $T_{\alpha}^{\prime} \in \mathbb{S} \cap V^{P_{\alpha}}$.

Lemma 2.3 Assume (**) and suppose $F=\left\{\alpha_{l}: l<m\right\} \subseteq \zeta$ is finite and enumerated in increasing order, and $p \in P_{\zeta}$, then there exists $q \leq p, n<\omega$, and $\left\langle Q_{l} \subseteq 2^{n}: l<m\right\rangle$ such that for each $l<m$

$$
q \Vdash “ T_{\alpha_{l}} \cap 2^{n}=\check{Q}_{l} \text { " }
$$

and for any $k<l<m$ if $s \in Q_{l} \cap Q_{k}$ then

$$
q \Vdash{ }^{*} T_{\alpha_{l} s} \subseteq T_{\alpha_{k}} \text { ". }
$$

proof:

Left to the reader.

Lemma 2.4 Assume (**) and suppose $\gamma \leq \min (H)$ where $H \subseteq \zeta$ is finite and $p \in P_{\zeta}$, then there exists $q_{0}, q_{1} \leq p$ with $q_{0}\left\lceil\gamma=q_{1} \uparrow \gamma\right.$ and disjoint $t_{0}, t_{1} \subseteq 2^{n}$ for some $n<\omega$ such that for each $\alpha \in H$ and $i=0,1$

$$
q_{i} \Vdash " T_{\alpha} \cap 2^{n} \subseteq \check{t}_{i} "
$$

proof:

It is easy to show:

Suppose $P_{1}, P_{2}$ are arbitrary posets and $T_{i}^{\prime} \in \mathbb{S} \cap V^{P_{i}}$. Given $p_{1} \in P_{1} * Q_{T_{1}^{\prime}}$ and $p_{2} \in P_{2} * Q_{T_{2}^{\prime}}$ there exists $n<\omega, q_{1} \leq p_{1}, q_{2} \leq p_{2}, t_{0} \subseteq 2^{n}, t_{1} \subseteq 2^{n}$ such that $t_{0} \cap t_{1}=\emptyset$ and for $i=0,1 \quad q_{i} \Vdash T_{i} \cap 2^{n} \subseteq \check{t}_{i}$.

The lemma follows by iteratively applying this statement to all pairs in $H$ with $V^{P_{\gamma}}$ as the ground model. 
Lemma 2.5 Assume (**) and suppose that $\zeta$ is a limit ordinal and for all $\alpha<\zeta$

$$
V^{P_{\alpha}} \models(*)\left\langle T_{j}: j<\alpha\right\rangle,
$$

then $V^{P_{\zeta}}=(*)\left\langle T_{j}: j<\zeta\right\rangle$.

proof:

Let

$$
p \vdash \text { " }\left\langle F_{\alpha}^{\prime}: \alpha<\omega_{1}\right\rangle \text { are pairwise disjoint finite subsets of } \zeta \text { ". }
$$

We must show there exists $q \leq p$ and $\alpha \neq \beta$ such that

$$
q \Vdash \forall i \in F_{\alpha}^{\prime} \forall j \in F_{\beta}^{\prime}\left[T_{i}\right] \cap\left[T_{j}\right]=\emptyset .
$$

Let $\left\langle p_{\alpha}, F_{\alpha}: \alpha<\omega_{1}\right\rangle \in V$ be such that $p_{\alpha} \leq p$ and

$$
p_{\alpha} \Vdash F_{\alpha}^{\prime}=\check{F}_{\alpha} .
$$

For some $A \in\left[\omega_{1}\right]^{\omega_{1}}\left\{F_{\alpha}: \alpha \in A\right\}$ forms a $\Delta$-system. The root of this $\Delta$-system must be empty, since $P_{\zeta}$ satisfies ccc.

Case 1. Cofinality of $\zeta$ is $\omega$.

For some $B \in[A]^{\omega_{1}}$, and $\gamma<\zeta$ we have that $F_{\alpha} \subseteq \gamma$ and $p_{\alpha} \in P_{\gamma}$ for every $\alpha \in B$. Let $G$ be a $P_{\gamma}$-filter such that $C=\left\{\alpha \in B: p_{\alpha} \in G\right\}$ is uncountable. ( Note that such a $G$ must exist, else there would exist a maximal antichain $Q$ such that for every $q \in Q$ there exists $\alpha<\omega_{1} q \Vdash C \subseteq \alpha$. Since $Q$ would be countable this would imply that $A$ is countable. ) Then by applying (*) in $V[G]$ there exists $\alpha \neq \beta \in C$ such that $\forall i \in F_{\alpha} \forall j \in F_{\beta}\left[T_{i}\right] \cap\left[T_{j}\right]=\emptyset$. Then $q \in G$ with $q \leq p_{\alpha}$ and $q \leq p_{\beta}$ is as required.

Case 2. Cofinality of $\zeta$ is $\omega_{1}$.

Apply Lemma 2.3. By cutting down to uncountable subset of $A$ we can assume that $\left|F_{\beta}\right|=m$ and $\left\langle Q_{l}: l<m\right\rangle$ are the same for each $\beta \in A$.

By passing to an uncountable subset of $A$ we can assume that there exists $\gamma<\zeta$ such that $F_{\alpha}=G_{\alpha} \cup H_{\alpha}$ where for each $\alpha \in A \quad G_{\alpha} \subseteq \gamma$ and $\left\langle\min \left(H_{\alpha}\right): \alpha \in A\right\rangle$ is unbounded in $\zeta$, and if $\alpha, \beta \in A$ and $\alpha<\beta$ then $\max \left(H_{\alpha}\right)<\min \left(H_{\beta}\right)$. Apply Lemma 2.4 to each $H_{\alpha}$ obtaining $q_{\alpha}^{i}$ for $i=0,1$ with

$$
q_{\alpha}={ }^{\text {def }} q_{\alpha}^{0}\left\lceil\min \left(H_{\alpha}\right)=q_{\alpha}^{1} \uparrow \min \left(H_{\alpha}\right)\right.
$$


and disjoint $t_{0}^{\alpha}, t_{1}^{\alpha}$. Again by passing to an uncountable subset we may assume $t_{0}, t_{1}$ are the same for each $\alpha \in A$. By cutting down $A$ and increasing $\gamma$ we may suppose that $\left\{\operatorname{support}\left(q_{\alpha}^{0}\right) \cup \operatorname{support}\left(q_{\alpha}^{1}\right): \alpha \in A\right\}$ is a $\Delta$-system whose root is a subset of $\gamma$. By the same argument as was used in Case 1, we can find distinct $\alpha, \beta \in A$ such that $q_{\alpha}$ and $q_{\beta}$ are compatible and

$$
\forall i \in G_{\alpha} \forall j \in G_{\beta}\left[T_{i}\right] \cap\left[T_{j}\right]=\emptyset .
$$

Claim 1. $q_{\alpha}^{0}$ and $q_{\beta}^{1}$ are compatible.

proof:

By definition $q_{\delta}=q_{\delta}^{i}\left\lceil\min \left(H_{\delta}\right)\right.$. Also $q_{\alpha}$ and $q_{\beta}$ are compatible elements of $P_{\gamma}$. Since the supports form a $\Delta$-system with root contained in $\gamma$ they are compatible.

Claim 2. $q_{\alpha}^{0} \cup q_{\beta}^{1} \Vdash \forall i \in F_{\alpha} \forall j \in F_{\beta}\left[T_{i}\right] \cap\left[T_{j}\right]=\emptyset$.

proof:

Case a. $i \in G_{\alpha}$ and $j \in G_{\beta}$.

This is true by the way we picked $\alpha$ and $\beta$.

Case b. $i \in H_{\alpha}$ and $j \in H_{\beta}$.

$q_{\alpha}^{0} \Vdash T_{i} \cap 2^{n} \subset t_{0}$ and $q_{\alpha}^{1} \Vdash T_{j} \cap 2^{n} \subset t_{1}$, but $t_{0} \cap t_{1}=\emptyset$.

Case c. $\alpha_{l}=i \in H_{\alpha}$ and $\beta_{k}=j \in G_{\beta}$.

Let $F_{\alpha}=\left\{\alpha_{l}: l<m\right\}$ and $F_{\beta}=\left\{\beta_{l}: l<m\right\}$, and so $k<l$. Note that

$$
q_{\alpha} \Vdash T_{\alpha_{l}} \cap 2^{n}=Q_{l}
$$

and

$$
q_{\beta} \Vdash T_{\alpha_{k}} \cap 2^{n}=Q_{k} .
$$

If $s \in Q_{l} \cap Q_{k}$, then

$$
q_{\alpha} \mid \vdash T_{\alpha_{l} s} \subseteq T_{\alpha_{k}}
$$

But by Case (a) we know

$$
q_{\alpha} \cup q_{\beta} \Vdash\left[T_{\alpha_{k}}\right] \cap\left[T_{\beta_{k}}\right]=\emptyset .
$$

Consequently $q_{\alpha} \cup q_{\beta} \Vdash\left[T_{\alpha_{l}}\right] \cap\left[T_{\beta_{k}}\right]=\emptyset$.

Case d. $\alpha_{l}=i \in G_{\alpha}$ and $\beta_{k}=j \in H_{\beta}$.

Same as Case (c). 


\section{Laver tree forcing}

Let $\mathbb{L}$ be Laver tree forcing, that is conditions are subtrees $p \subseteq \omega^{<\omega}$ with the property that there exists a node $s \in p$ called its root such that:

1. for every $t \in p$ either $t \subseteq s$ or $s \subseteq t$,

2. for every $t \in p$ if $s \subseteq t$, then the set $\operatorname{split}(p, t)={ }^{\operatorname{def}}\left\{n \in \omega: t^{\wedge} n \in p\right\}$ is infinite.

Theorem 3.1 Assume MA. Then $\mathbb{L}$ does not collapse cardinals.

Given $A=\left\langle A_{s} \in[\omega]^{\omega}: s \in \omega^{<\omega}\right\rangle$ and $s \in \omega^{<\omega}$ define $p_{s}(A)=p \in \mathbb{L}$ to be the unique Laver tree such that the root of $p$ is $s$ and for every $t \supseteq s$ with $t \in p$ we have that $\operatorname{split}(p, t)=A_{t}$.

Lemma 3.1 Suppose $\vdash \tau \in V$ and $B=\left\langle B_{s}: s \in \omega^{<\omega}\right\rangle$ where each $B_{s} \in$ $[\omega]^{\omega}$. Then there exists a countable $X$ and $A=\left\langle A_{s} \in\left[B_{s}\right]^{\omega}: s \in \omega^{<\omega}\right\rangle$ such that for every $s \in \omega^{<\omega}$

$$
p_{s}(A) \Vdash \tau \in X \text {. }
$$

proof:

Laver proved that for any $p \in \mathbb{L}$ there exists $q \leq p$ with the same root and $X$ countable such that

$$
q \Vdash \tau \in X \text {. }
$$

Build $p_{n} \in \mathbb{L}$ as follows. At stage $n$, let $s$ be the $n^{\text {th }}$ element of $\omega^{<\omega}$. If $s \in p_{m}$ for some $m<n$, then do nothing. Otherwise, take $p_{n} \leq p_{s}(B)$ and $X_{n}$ countable such that $s$ is the root of $p_{n}$ and $p_{n} \Vdash \tau \in X_{n}$. For every $t \in p_{n}$ such that $s \subseteq t$, let $A_{t}=\operatorname{split}\left(p_{n}, t\right)$. Finally let $X=\bigcup_{n<\omega} X_{n}$.

The next lemma proves the theorem.

Lemma 3.2 Suppose $M A, \kappa<\mathfrak{c}$, and $p \Vdash \tau: \kappa \rightarrow V$, then there exist $q \leq p$ and $\left\langle X_{\alpha}: \alpha<\kappa\right\rangle$ such that for every $\alpha \quad X_{\alpha}$ is countable and $q \Vdash \tau(\alpha) \in \check{X}_{\alpha}$. proof:

To simplify notation let $p=\omega^{<\omega}$.

Let $Q=\left\{\left\langle A_{s}: s \in \omega^{<\omega}\right\rangle: A_{s} \in[\omega]^{\omega}\right\}$ and for $A, B \in Q$ define $A \subseteq^{*} B$ iff for all $s \in \omega^{<\omega} \quad A_{s} \backslash B_{s}$ is finite.

Build $A_{\alpha} \in Q$ for $\alpha<\kappa$ such that 
1. $\alpha<\beta$ implies $A_{\beta} \subseteq^{*} A_{\alpha}$.

2. there exists a countable set $X_{\alpha}$ such that for every $s \in \omega^{<\omega}$

$$
p_{s}\left(A_{\alpha}\right) \Vdash \tau(\alpha) \in X_{\alpha} .
$$

At stage $\alpha$ use the MA to get $A \in Q$ such that for all $\beta<\alpha A \subseteq^{*} A_{\beta}$. (This is a well known consequence of Martin's Axiom, apply Solovay's Lemma, Kunen [21] p.57, to each of the families $\left\{A_{\beta s}: \beta<\alpha\right\}$ for $s \in \omega^{<\omega}$.) Then use Lemma 3.1 to get $A_{\alpha} \subseteq^{*} A$ as desired.

Now consider the following poset:

$$
\mathbb{P}=\left\{(T, W): T \text { is a finite subtree of } \omega^{<\omega}, W \in[\kappa]^{<\omega}\right\}
$$

Order $\mathbb{P}$ by $(T, W) \leq\left(T^{\prime}, W^{\prime}\right)$ iff

1. $W \supseteq W^{\prime}, T \supseteq T^{\prime}$, and

2. $\forall n<\omega \forall s \in\left(T \backslash T^{\prime}\right) \cap \omega^{n+1} \forall \alpha \in W^{\prime} s(n) \in A_{\alpha, s}$.

Since any two conditions with the same $T$ part are compatible, $\mathbb{P}$ is $\sigma$ centered, so we can apply MA to it. For $G$ a $\mathbb{P}$-filter let

$$
q=\cup\{T: \exists W(T, W) \in G\} .
$$

If $G$ meets the dense subsets of $\mathbb{P}$ of the form

$$
D_{s, n}=\left\{p \in \mathbb{P}: p \Vdash s \notin q \text { or } \exists m>n s^{\wedge} m \in T_{p}\right\}
$$

then we will have that $q \in \mathbb{L}$ with the empty sequence as its root. For any $\alpha<\kappa$ let

$$
D_{\alpha}=\left\{p \in \mathbb{P}: \alpha \in W_{p}\right\}
$$

Each $D_{\alpha}$ is dense.

Hence by MA we can get $q \in \mathbb{L}$ with the empty node as root such that for every $\alpha<\kappa$ there is finite subtree $T \subseteq q$ such that for every $t \in q \backslash T$ we have $t \in p_{t \uparrow(|t|-1)}\left(A_{\alpha}\right)$. This implies that for every $r \leq q$ there exists $s$ such that $p_{s}\left(A_{\alpha}\right)$ is compatible with $r$. It follows that

$$
q \Vdash \forall \alpha<\kappa \tau(\alpha) \in X_{\alpha}
$$




\section{Superperfect trees}

Superperfect tree forcing $\mathbb{F}$ is defined as follows. For $p$ a subtree of $\omega^{<\omega}$ define the splitting nodes of $p$ :

$$
\operatorname{splitnodes}(p)=\left\{s \in p: \exists^{\infty} n \in \omega s^{\wedge} n \in p\right\} .
$$

Define $p \in \mathbb{F}$ iff

1. $p$ is a nonempty subtree of $\omega^{<\omega}$,

2. $\operatorname{splitnodes}(p)$ is dense in $p$, ie $\forall s \in p \exists t \in \operatorname{splitnodes}(p)$ with $t \supseteq s$, and

3. if any node in $p$ splits it is a splitting node, ie if there exists more than one $n \in \omega$ such that $s^{\wedge} n \in p$, then there are infinitely many $n \in \omega$ such that $s^{\wedge} n \in p$.

Theorem 4.1 Assume MA. Then $\mathbb{F}$ does not collapse cardinals. ${ }^{3}$

proof:

Call a sequence $\left\langle P_{s}: s \in \omega^{<\omega}\right\rangle$ good iff

1. each $P_{s} \subseteq \omega^{<\omega}$ is infinite,

2. $t \in P_{s}$ implies $s \varsubsetneqq t$, and

3. for $s \in \omega^{n}$ if $t, t^{\prime} \in P_{s}$ and $t \neq t^{\prime}$, then $t(n) \neq t^{\prime}(n)$.

Given any good sequence $\left\langle P_{s}: s \in \omega^{<\omega}\right\rangle$ we determine $\left\langle p_{s} \in \mathbb{F}: s \in \omega^{<\omega}\right\rangle$ as follows. For each $s$ let $S$ be is smallest subset of $\omega^{<\omega}$ such that $P_{s} \subseteq S$ and if $t \in S$ then $P_{t} \subseteq S$. Then $p_{s}$ is the unique condition in $\mathbb{F}$ such that $S=\operatorname{splitnodes}\left(p_{s}\right)$. In other words, $P_{s}$ says that $s$ is a splitting node and the splitting nodes immediately below $s$ are $P_{s}$. Define $\left\langle P_{s}: s \in \omega^{<\omega}\right\rangle \leq$ $\left\langle Q_{s}: s \in \omega^{<\omega}\right\rangle$ iff $p_{s} \subseteq q_{s}$ for each $s \in \omega^{<\omega}$. An equivalent definition would be for each $s \in \omega^{<\omega}$ and $t \in P_{s}$ there exists $k$ and $s_{0}, s_{1}, \ldots, s_{k}$ where $s=s_{0} \subseteq s_{1} \subseteq \ldots \subseteq s_{k}=t s_{i+1} \in Q_{s_{i}}$ for $i=0,1, \ldots, k-1$.

\footnotetext{
${ }^{3}$ Added post publication: Goldstern, Roslanowski, and Spinas found a mistake in this proof. The relation $\leq^{*}$ is not transitive. A correct proof is given in the paper: Goldstern, Martin; Johnson, Mark J.; Spinas, Otmar Towers on trees. Proc. Amer. Math. Soc. 122 (1994), no. 2, 557-564.
} 
Lemma 4.1 Given $\tau$ such that $\vdash_{\mathbb{F}}$ " $\tau \in V$ ", and good $\left\langle P_{s}: s \in \omega^{<\omega}\right\rangle$ there exists a good $\left\langle Q_{s}: s \in \omega^{<\omega}\right\rangle \leq\left\langle P_{s}: s \in \omega^{<\omega}\right\rangle$ and a countable set $\Sigma$ such that for every $s \in \omega^{<\omega} q_{s} \Vdash$ " $\tau \in \Sigma$ ".

proof:

For any $p \in \mathbb{F}$ with smallest splitting node $s$ (ie root) there exists $q \subseteq p$ such that $s \in \operatorname{splitnodes}(q)$ and a countable $\Sigma$ such that $q \vdash^{\prime \prime} \tau \in \Sigma$ ". Now just apply this fact repeatedly down the $s \in \omega^{<\omega}$.

Define $\left\langle P_{s}: s \in \omega^{<\omega}\right\rangle \leq^{*}\left\langle Q_{s}: s \in \omega^{<\omega}\right\rangle$ iff there exists $\left\langle P_{s}^{\prime}: s \in \omega^{<\omega}\right\rangle \leq$ $\left\langle Q_{s}: s \in \omega^{<\omega}\right\rangle$ such that for every $s \in \omega^{<\omega} P_{s}={ }^{*} P_{s}^{\prime}$ (equal mod finite). Similarly for $p, q \in \mathbb{F}$ define $p \leq^{*} q$ iff there exists $f: \operatorname{splitnodes}(p) \rightarrow \omega$ such that $p_{f} \subseteq q$, where

$$
p_{f}=p \backslash\left\{r \in \omega^{<\omega}: \exists s \in \operatorname{splitnodes}(p) \exists n<f(s) s^{\wedge} n \subseteq r\right\} .
$$

We think of $p_{f}$ as being obtained from $p$ by pruning finitely many nodes from beneath each splitting node of $p$. Note that $p_{f} \in \mathbb{F}$.

Lemma 4.2 The following are equivalent:

1. $\left\langle P_{s}: s \in \omega^{<\omega}\right\rangle \leq^{*}\left\langle Q_{s}: s \in \omega^{<\omega}\right\rangle$

2. for every $s \in \omega^{<\omega}, p_{s} \leq^{*} q_{s}$.

proof:

Left to reader.

Lemma 4.3 (MA) Suppose $\gamma<\mathfrak{c}$ and $\left\langle P_{s}^{\alpha}: s \in \omega^{<\omega}\right\rangle$ for $\alpha<\gamma$ are good and have the property that $\alpha>\beta$ implies $\left\langle P_{s}^{\alpha}: s \in \omega^{<\omega}\right\rangle \leq^{*}\left\langle P_{s}^{\beta}: s \in \omega^{<\omega}\right\rangle$. Then there exists a good $\left\langle P_{s}: s \in \omega^{<\omega}\right\rangle$ such that for every $\alpha<\gamma \quad\left\langle P_{s}: s \in\right.$ $\left.\omega^{<\omega}\right\rangle \leq^{*}\left\langle P_{s}^{\alpha}: s \in \omega^{<\omega}\right\rangle$.

proof:

Let $Q$ be the following poset, $\left(A_{s}: s \in F, H\right) \in Q$ iff

1. $H \subseteq \gamma, F \subseteq \omega^{<\omega}, A_{s} \subseteq \omega^{<\omega}$ for $s \in F$ are all finite,

2. $t \in A_{s}$ implies $s \varsubsetneqq t$, and 
3. $t, t^{\prime} \in A_{s}$ and $t \neq t^{\prime}$ implies $t(n) \neq t^{\prime}(n)$, where $s \in \omega^{n}$.

We define $\left(\hat{A}_{s}: s \in \hat{F}, \hat{H}\right) \leq\left(A_{s}: s \in F, H\right)$ iff

1. $\hat{F} \supseteq F, \hat{H} \supseteq H, \hat{A}_{s} \supseteq A_{s}$ for $s \in F$, and

2. for each $s \in F$ if $t \in \hat{A}_{s} \backslash A_{s}$ and $\alpha \in H$, then $t \in \operatorname{splitnodes}\left(p_{s}^{\alpha}\right)$.

Note that $Q$ is ccc, in fact $\sigma$-centered, since $\left(A_{s}: s \in F, H \cup \hat{H}\right)$ extends both $\left(A_{s}: s \in F, H\right)$ and $\left(A_{s}: s \in F, \hat{H}\right)$. For any $\alpha<\gamma$

$$
\left\{\left(A_{s}: s \in F, H\right) \in Q: \alpha \in H\right\}
$$

is dense in $Q$, since $\left(A_{s}: s \in F, H \cup\{\alpha\}\right) \leq\left(A_{s}: s \in F, H\right)$. In order to quarantee that $A_{t}$ grows up into an infinite set, we need only check that the following sets are dense. Fix $t \in \omega^{n}$ and $m<\omega$ and define

$$
E_{t, m}=\left\{\left(A_{s}: s \in F, H\right) \in Q: t \in F, \exists r \in A_{t} \quad r(n)>m\right\}
$$

To check this let $\left(A_{s}: s \in F, H\right) \in Q$ and put $t$ into $F$ by letting $A_{t}=\emptyset$ if neccessary. Let $\alpha=\max \{H\}$ and let $p=p_{s}^{\alpha}$. Then there exists $f$ : $\operatorname{splitnodes}(p) \rightarrow \omega$ such that for every $\beta \in H$ we have $p_{f} \subseteq p_{s}^{\beta}$. Consequently any $r \in \operatorname{splitnodes}\left(p_{f}\right)$ with $r \supset s$ and $r(n)>m$ can be added to $A_{t}$. Finally if $G$ is sufficiently $Q$-generic, then $\left\langle P_{s}: s \in \omega^{<\omega}\right\rangle$ defined by

$$
P_{t}=\bigcup\left\{A_{t}: \exists\left(A_{s}: s \in F, H\right) \in G, t \in F\right\}
$$

is as required.

Lemma 4.4 (MA) Suppose $\kappa<\mathfrak{c}$ is an uncountable regular cardinal and $p_{\alpha} \in \mathbb{F}$ for $\alpha<\kappa$ and $r \in \mathbb{F}$ have the property that for every $\alpha<\kappa r \leq^{*} p_{\alpha}$. Then there exists $q \subseteq r$ and $\Gamma \in[\kappa]^{\kappa}$ such that $q \subseteq p_{\alpha}$ for every $\alpha \in \Gamma$.

proof:

For each $\alpha<\kappa$ let $f_{\alpha}: \operatorname{splitnodes}(r) \rightarrow \omega$ be such that $r_{f_{\alpha}} \subseteq p_{\alpha}$ where as before,

$$
r_{f}=r \backslash\left\{t \in \omega^{<\omega}: \exists s \in \operatorname{splitnodes}(f) \exists m<f(s) ; s^{\wedge} m \subset t\right\}
$$

that is $f_{\alpha}(s)$ tells what finite set of nodes below the splitting node $s$ of $r$ we should prune from $r$ so as to end up with subtree of $p_{\alpha}$. It is well known that 
MA gives us $f: \operatorname{splitnodes}(r) \rightarrow \omega$ that for all $\alpha<\kappa$ for all but finitely many $s \in \operatorname{splitnodes}(r) \quad f_{\alpha}(s)<f(s)$. By changing $f$ on a finite set we can find $\Gamma \in[\kappa]^{\kappa}$ such that for all $\alpha \in \Gamma$ and for all $s \in \operatorname{splitnodes}(r) f_{\alpha}(s)<f(s)$. It follows from this that $q=r_{f} \subseteq p_{\alpha}$ for all $\alpha \in \Gamma$

Proof of Theorem 4.1: Suppose that forcing with $\mathbb{F}$ did collapse cardinals, then there would exist regular cardinals $\kappa<\lambda \leq \mathfrak{c}, p \in \mathbb{F}$ and a name $\tau$ such that

$$
p \Vdash \tau: \kappa \rightarrow \lambda \text { is increasing and cofinal. }
$$

To simplyfy notation assume $p=\omega^{<\omega}$. Using Lemma 4.1 and Lemma 4.3 build a sequence of good $\left\langle P_{s}^{\alpha}: s \in \omega^{<\omega}\right\rangle$ for $\alpha<\kappa$ such that

1. $\alpha>\beta$ implies $\left\langle P_{s}^{\alpha}: s \in \omega^{<\omega}\right\rangle \leq^{*}\left\langle P_{s}^{\beta}: s \in \omega^{<\omega}\right\rangle$ and

2. for any $\alpha$ there exists a countable $\Sigma$ such that for every $s \in \omega^{<\omega}$ $p_{s}^{\alpha} \vdash^{\prime \prime} \tau(\alpha) \in \Sigma$ ".

Use Lemma 4.3 one more time to obtain $\left\langle R_{s}: s \in \omega^{<\omega}\right\rangle$ such that $\left\langle R_{s}\right.$ : $\left.s \in \omega^{<\omega}\right\rangle \leq^{*}\left\langle P_{s}^{\alpha}: s \in \omega^{<\omega}\right\rangle$ for each $\alpha<\kappa$. Fix $s$ (say the empty node) and define $r=r_{s}$ and $p_{\alpha}=p_{s}^{\alpha}$. Since these satisfy the hypothesis of Lemma 4.4 we can obtain $q \subseteq r$ and $\Gamma \in[\kappa]^{\kappa}$ such that $q \subseteq p_{\alpha}$ for every $\alpha \in \Gamma$. But now there exists countable sets $\left\{\Sigma_{\alpha}: \alpha \in \Gamma\right\}$ such that

$$
q \Vdash \forall \alpha \in \Gamma \tau(\alpha) \in \Sigma_{\alpha}
$$

which means that the range of $\tau$ cannot be cofinal in $\lambda$.

\section{References}

[1] U.Abraham, A minimal model for $\neg \mathrm{CH}$ : iteration of Jensen's reals, Transactions of the American Mathematical Society, 281(1984), 657674.

[2] U.Abraham, M.Rubin, S.Shelah, On the consistency of some partition theorems for continuous colorings and the structure of $\aleph_{1}$-dense real order types, Annals of Pure and Applied Logic, 29(1985), 123-206. 
[3] U.Abraham, S.Todorcevic, Martin's axiom and first-countable S- and Lspaces, in Handbook of set theoretic topology, edited by K.Kunen and J.E.Vaughan, North-Holland, (1984), 327-346.

[4] B.Aniszczyk,R.Frankiewicz,S.Plewik, Remarks on (s) and Ramseymeasurable functions, Bulletin of the Polish Academy of Sciences Mathematics, 35(1987), 479-485.

[5] B.Aniszczyk, Remarks on $\sigma$-algebra of $(s)$-measurable sets, Bulletin of the Polish Academy of Sciences Mathematics, 35(1987), 561-563.

[6] B.Balcar and P.Vojtas, Refining systems on boolean algebras, Lecture Notes in Mathematics, Springer-Verlag, 619(1977), 45-68.

[7] J.Baumgartner, Iterated forcing, in Surveys in Set Theory, edited by ARD Mathias, London Mathematical Society Lecture Note Series, 87(1983), 1-59.

[8] J.Baumgartner and R.Laver, Iterated perfect set forcing, Annals of Mathematical Logic, 17(1979), 271-288.

[9] A.Blass and S.Shelah, Near coherence of filters III: a simplified consistency proof,

[10] A.Blass, Applications of superperfect forcing and its relatives, in Set theory and its applications, York University 1987, Springer-Verlag Lecture Notes in Mathematics, 1401(1989), 18-40.

[11] J.B.Brown, Singular sets and Baire order, Real Analysis Exchange, (1985).

[12] J.B.Brown, Marczewski Null Sets and Intermediate Baire Order, Contemporary Mathematics, 94(1989), 43-50.

[13] J.B.Brown, The Ramsey sets and related sigma algebras and ideals,

[14] J.B.Brown and G.V.Cox, Classical theory of totally imperfect sets, Real Analysis Exchange, 7(1982), no2.

[15] J.B.Brown and K.Prikry, Variations on Lusin's Theorem, Transactions of the American Mathematical Society, 302(1987), 77-86 
[16] P.Corazza, The generalized Borel conjecture and strongly proper orders, Transactions of the American Mathematical Society, 316(1989), 115-140.

[17] K.Devlin, $\aleph_{1}$-trees, Annals of Mathematical Logic, 13(1978), 267-330.

[18] Y.Gurevich and S.Shelah, Monadic theory of order and topology in ZFC, Annals of Mathematical Logic, 23(1982), 179-198.

[19] H.Judah and S.Shelah, The Kunen-Miller chart, Journal of Symbolic Logic, 55(1990), 909-927.

[20] A.Kechris, On a notion of smallness for subsets of the Baire space, Transactions of the American Mathematical Society, 229(1977), 191-207.

[21] K.Kunen, Set Theory , North-Holland, 1980.

[22] K.Kunen, Where MA first fails, Journal of Symbolic Logic, 53(1988), 429-433.

[23] R.Laver, On the consistency of Borel's conjecture, Acta Mathematica., 137(1976), 151-169.

[24] E.Marczewski, Sur une classe de fonctions de W.Sierpiński et la classe correspondante d'ensembles, Fundamenta Mathematicae, 24(1935), 1734 .

[25] P.Matet,

[26] A.R.D.Mathias, "Happy Families", Annals of Mathematical Logic, 12(1977),59-111.

[27] A.Miller, Rational perfect set forcing, Contemporary Mathematics (American Mathematical Society), 31(1984), 143-159.

[28] A.Miller, Special subsets of the real line, in Handbook of Set Theoretic Topology, ed. by K.Kunen and J.Vaughan, North-Holland, 1984, 201-234.

[29] J.C.Morgan II, On the general theory of point sets II, Real Analysis Exchange, 12(1986), 377-386.

[30] J.Mycielski, Some new ideals of subsets on the real line, Colloquium Mathematicum, 20(1969), 71-76. 
[31] J.Pawlikowski, Parametrized Ellentuck Theorem, Journal of Combinatorial Theory Series B, 1990, ???

[32] G.E.Sacks, Forcing with perfect closed sets, Axiomatic Set Theory, ed by D.Scott, Proc. Sympos. Pure Math. Amer. Math. Soc., 13(1971), 331-355.

[33] S.Shelah, A weak generalization of MA to higher cardinals, Israel Journal of Mathematics, 30(1978), 297-306.

[34] J.Silver, Every analytic set is Ramsey, Journal of Symbolic Logic, 35(1970), 60-64.

[35] J.Steprans, The covering number of the Mycielski ideal,

[36] B.Velickovic, CCC posets of perfect trees,

[37] W.Weiss, Versions of Martin's Axiom, in Handbook of set theoretic topology, edited by K.Kunen and J.E.Vaughan, North-Holland, (1984), 827-886.

\section{Addresses}

Haim Judah, Department of Mathematics, Bar-Ilan University, 52900 Ramat-Gan, Israel, judah@bimacs.biu.AC.IL

Arnold W. Miller, Department of Mathematics, University of Wisconsin, 480 Lincoln Drive, Madison, WI, 53706, USA, miller@math.wisc.edu

Saharon Shelah, Institute of Mathematics, Hebrew University, Jerusalem, Israel

\section{Appendix: Stationary subsets of $\omega_{2}$}

In this appendix we solve a problem posed by M. Foreman: Does forcing with $\mathbb{S}$ add a stationary subset of $\omega_{2}$ which does not contain a stationary subset of the ground model?

Note that if $\omega_{2}$ is collapsed to $\omega_{1}$, then there exists a club subset of $\omega_{2}$ which does not contain an unbounded subset of the ground model. If $\mathfrak{c}=\omega_{1}$, then every new stationary subset $S$ of $\omega_{2}$ contains an old stationary set. This is because $\mathbb{S}$ has cardinality $\omega_{1}$ and so some $p \in \mathbb{S}$ must force stationarily 
many $\alpha \in S$. Amoeba Sacks forcing is defined by $\mathbb{P}=\{(p, n): n<\omega, p \in \mathbb{S}\}$ where $(p, n) \leq(q, m)$ iff $p \subseteq q, n \geq m$, and $p \cap 2^{m}=q \cap 2^{m}$. It is proper. If $\mathfrak{c}>\omega_{2}$ and MA(Amoeba Sacks), then every stationary subset of $\omega_{2}$ contains a ground model stationary set. To see this note that if

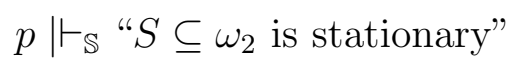

then by using Amoeba Sacks forcing we can find $q \leq p$ such that for every $\alpha<\omega_{2}$ there exists $n<\omega$ such that for every $s \in 2^{n} \cap q$ either $q_{s} \Vdash \alpha \in S$ or $q_{s} \Vdash \alpha \notin S$. Hence there must be some $s \in q$ such that

$$
\left\{\alpha<\omega_{2}: q_{s} \mid \vdash \alpha \in S\right\}
$$

is stationary.

Theorem 1 Suppose $\mathfrak{c}=\omega_{2}$ then forcing with $\mathbb{S}$ adds a stationary subset of $\omega_{2}$ that does not contain a stationary set from the ground model.

proof:

Let $\mathbb{S}=\left\{q_{\alpha}: \alpha<\omega_{2}\right\}$. Use Lemma 2 to obtain for each $\beta<\omega_{2}$ an antichain $\left\{p_{\beta \alpha} \leq q_{\alpha}: \alpha<\beta\right\}$. Let $p_{\beta \alpha}^{i} \leq p_{\beta \alpha}$ for $i=0,1$ be two incompatible extensions.

Now let $S=\left\{\left\langle p_{\beta \alpha}^{1}, \check{\beta}\right\rangle: \alpha<\beta<\omega_{2}\right\}$. Then the following two facts hold:

1. $\vdash_{\mathbb{S}} S \subseteq \check{\omega}_{2}$

2. for every $p \in \mathbb{S}\left\{\alpha<\omega_{2}: p \mid \vdash \alpha \in S\right.$ or $\left.p \Vdash \alpha \notin S\right\}$ is bounded in $\omega_{2}$.

Thus neither $S$ nor its complement can contain a stationary set (or even an unbounded subset of $\omega_{2}$ ) which is in the ground model. Since one of the two must be stationary the theorem is proved.

Lemma 2 Suppose $\mathfrak{c}=\omega_{2}$ and $Q=\left\{q_{\alpha}: \alpha<\omega_{1}\right\} \subseteq \mathbb{S}$, then there exists an antichain $\left\{p_{\alpha}: \alpha<\omega_{1}\right\}$ such that for each $\alpha<\omega_{1} p_{\alpha} \leq q_{\alpha}$.

proof:

Inductively construct $p_{\alpha}$ so that for every $\beta>\alpha\left[p_{\alpha}\right] \cap\left[q_{\beta}\right]$ is nowhere dense in $\left[q_{\beta}\right]$. This is easy to do. At stage $\alpha$, find $r \in \mathbb{S}$ such that

$$
[r] \subseteq\left(\left[q_{\alpha}\right] \backslash \bigcup_{\beta<\alpha}\left[p_{\beta}\right]\right) .
$$


Then split $[r]$ into $\omega_{2}$ perfect disjoint sets. Note that for any $\beta>\alpha$ at most countably many of these perfect sets can fail to be nowhere dense in $\left[q_{\beta}\right]$. Let $p_{\alpha}$ be any of the remaining ones.

By a theorem of Balcar and Vojtas [6], Lemma 2 is true in much more generality. All that is really needed is: every $p \in \mathbb{P}$ has $\omega_{2}$ incompatible extensions. Consequently the result holds for all of the standard tree kinds of forcing, eg Laver, Mathias, Silver, etc. 